\title{
Autophagy pathway upregulation in a human iPSC-derived neuronal model of Cohen syndrome with VPS13B missense mutations
}

You-Kyung Lee ${ }^{1}$, Soo-Kyeong Lee ${ }^{1}$, Suin Choi ${ }^{1,2}$, Yang Hoon Huh², Ji-Hye Kwak ${ }^{3}$, Yong-Seok Lee ${ }^{4}$, Deok-Jin Jang ${ }^{5}$, Jae-Hyung Lee ${ }^{6}$, Kyungmin Lee ${ }^{3}$, Bong-Kiun Kaang ${ }^{7}$, Chae-Seok Lim ${ }^{8^{*}}$ and Jin-A Lee ${ }^{1^{*}}$ (D)

\begin{abstract}
Significant clinical symptoms of Cohen syndrome (CS), a rare autosomal recessive disorder, include intellectual disability, facial dysmorphism, postnatal microcephaly, retinal dystrophy, and intermittent neutropenia. CS has been associated with mutations in the VPS13B (vacuolar protein sorting 13 homolog B) gene, which regulates vesiclemediated protein sorting and transport; however, the cellular mechanism underlying CS pathogenesis in patientderived neurons remains uncertain. This report states that autophagic vacuoles accumulate in CS fibroblasts and the axonal terminals of CS patient-specific induced pluripotent stem cells (CS IPSC)-derived neurons; additionally, autophagic flux was significantly increased in CS-derived neurons compared to control neurons. VPS13B knockout HeLa cell lines generated using the CRISPR/Cas9 genome editing system showed significant upregulation of autophagic flux, indicating that VSP13B may be associated with autophagy in CS. Transcriptomic analysis focusing on the autophagy pathway revealed that genes associated with autophagosome organization were dysregulated in CS-derived neurons. ATG4C is a mammalian ATG4 paralog and a crucial regulatory component of the autophagosome biogenesis/recycling pathway. ATG4C was significantly upregulated in CS-derived neurons, indicating that autophagy is upregulated in CS neurons. The autophagy pathway in CS neurons may be associated with the pathophysiology exhibited in the neural network of CS patients.
\end{abstract}

Keywords: Cohen syndrome, Autophagy, VPS13B, iPSC

\section{Main text}

Cohen syndrome (CS) is a rare congenital, neurodevelopmental disorder that manifests clinical features such as obesity, hypotonia, intellectual disabilities, microcephaly, and distinctive facial features [1]. Many people with CS have autosomal recessive mutations in the VPS13B

\footnotetext{
* Correspondence: cslimwk1@wku.ac.kr; leeja@hnu.kr

${ }^{8}$ Department of Pharmacology, Wonkwang University School of Medicine, 460 Iksan-daero, Iksan 54538, Korea

'Department of Biological Sciences and Biotechnology, Hannam University, 1646 Yuseongdaero, Yuseong-gu, Daejeon 34054, Korea

Full list of author information is available at the end of the article
}

gene (also known as COH1), which harbors up to 62 exons, and an open reading frame encoding 4022 amino acids and is generally involved in vesicle-mediated trafficking and intracellular protein transport [2]. While VPS13B mutant CS mouse models exhibited specific clinical symptoms in CS [3], there is a growing need for pathophysiological studies in human cells derived from CS patient-specific induced pluripotent stem cells (iPSCs). Human skin fibroblasts from CS patients, carrying frameshift or nonsense mutations in VPS13B, display a fragmented Golgi complex that leads to disruption of Golgi integrity [4]. 
To uncover pathological phenotypes at the ultrastructural level in CS patients with VPS13B novel mutations, we performed electron microscopic (EM) analysis on control and CS fibroblasts [5]. There were no gross defects regarding the morphology of the nuclei, ER, or mitochondria (including the Golgi-complex at the ultrastructural level) in CS fibroblasts, however, autophagic vacuoles (AVs), which are a hallmark of autophagy, accumulated tremendously in the cytosol of CS fibroblasts when compared to control fibroblasts (Fig. 1a). The number of early autophagosomes or autolysosomes was significantly increased in CS fibroblasts compared to control fibroblasts (Fig. 1b). To further examine autophagic activity in control and CS fibroblasts, autophagic flux assays were performed in the presence or absence of lysosomal inhibitors (100 nM chloroquine or $100 \mathrm{nM}$
BafA1) for $24 \mathrm{~h}$ with or without rapamycin (100 $\mathrm{nM})$. In the presence of lysosomal inhibitors, more accumulation of LC3B-II was found in CS fibroblasts compared to control fibroblasts, indicating that CS fibroblasts exhibit upregulated autophagic flux (Fig. 1c-d).

The autophagy pathway is a conserved cellular degradation and recycling system that relies on lysosomal degradation of superfluous or cytotoxic components and damaged organelles engulfed by autophagic vacuoles in a selective or non-selective manner $[6,7]$. Increasing evidence suggests that neuronal presynaptic autophagy in the axon terminal is essential for synaptic maintenance and plasticity [8]. To investigate whether the autophagy pathway is upregulated in CS iPSC-derived neurons, which previously showed synaptic protein (e.g., SV2B) alteration, 4-week old CS neurons were analyzed at the
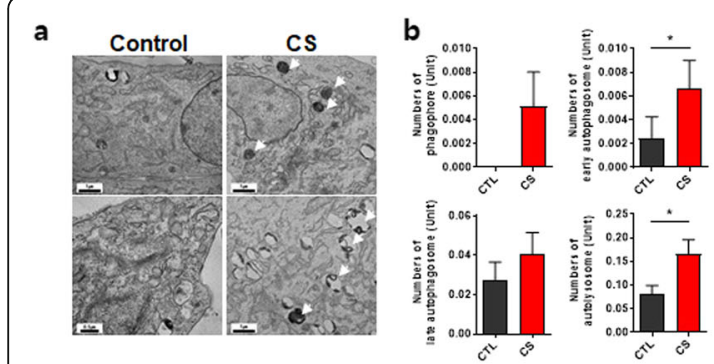

今 $0^{5}$
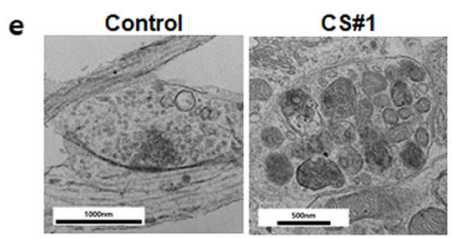

h
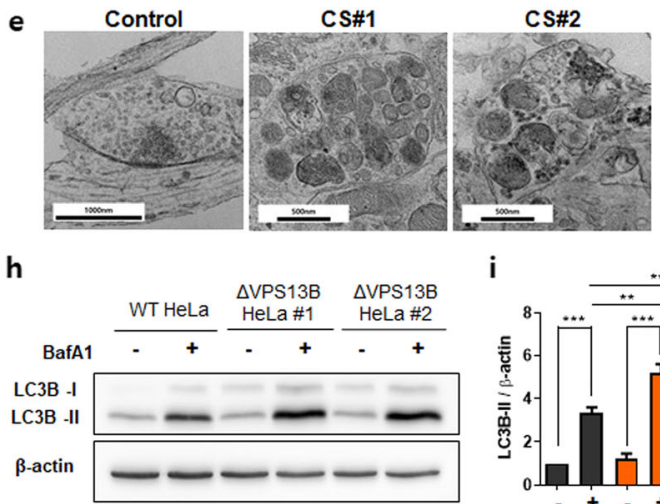

i

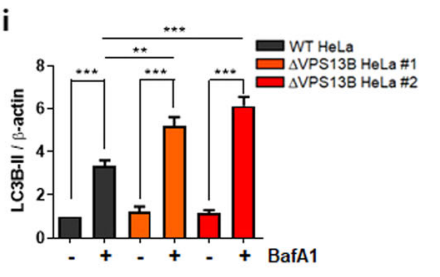

c

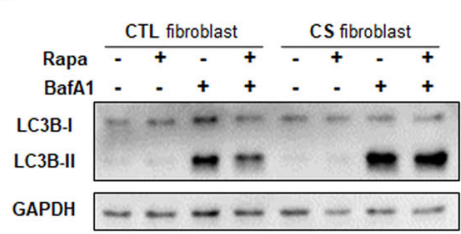

f

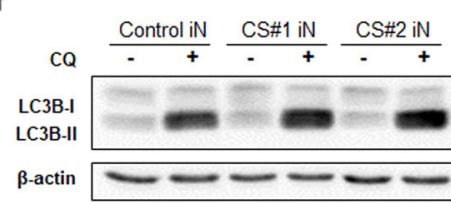

j

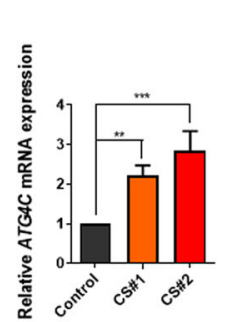

d

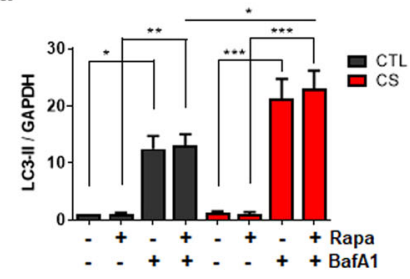

g

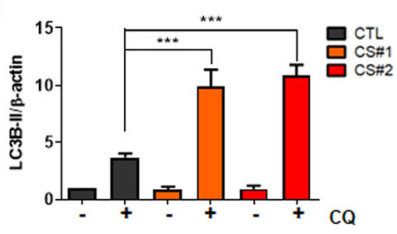

k
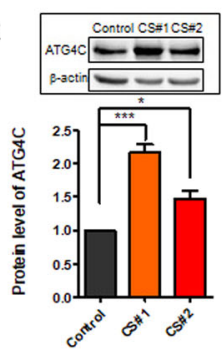

Fig. 1 Autophagic flux is upregulated in Cohen syndrome (CS) fibroblasts and CS iPSC-derived neurons. a Electron microscopic images of control and CS fibroblasts. b Quantitative analysis of EM images. The bar graph indicates the means \pm SEM. *, $p<0.05$ (Student's t-test). $\mathbf{c}$ Western blot analysis showing autophagic flux in the presence or absence of a lysosomal inhibitor (bafilomycin A1 [BafA1], 100 nM) with or without rapamycin (100 nM, 4 h) using anti-LC3 or -GAPDH antibodies. d Relative expression of LC3-II normalized by GAPDH. The bar graph indicates the means \pm SEM from three independent experiments. ${ }^{*}, p<0.05 ;{ }^{* *}, p<0.01 ;{ }^{* * *}, p<0.001$ (one-way ANOVA followed by Tukey's Multiple Comparison Test). e Electron microscopic images of synaptic regions in control and CS iPSC-derived neurons. $\mathbf{f}$ Western blot analysis showing autophagic flux in the presence or absence of a lysosomal inhibitor (chloroquine [CQ], $100 \mathrm{nM}$ ) using anti-LC3 or - $\beta$-actin antibodies. $\mathbf{g}$ Relative expression of LC3-II normalized by $\beta$-actin. The bar graph indicates the means \pm SEM from three independent experiments. ${ }^{* * *}, p<0.001$ (one-way ANOVA followed by Tukey's Multiple Comparison Test). $\mathbf{h}$ Western blot analysis showing autophagic flux in the presence or absence of a lysosomal inhibitor (BafA1, $100 \mathrm{nM}$ ) using anti-LC3 or - $\beta$-actin antibodies with WT or VPS13B KO HeLa cell (\#1, or \#2) lysates. i Quantification of band intensity of LC3II or $\beta$-actin. The bar graph indicates the means \pm SEM from three independent experiments. ${ }^{* *}, p<0.01 ;{ }^{* * *}, p<0.001$ (one-way ANOVA followed by Tukey's Multiple Comparison Test). $\mathbf{j}$ The mRNA level of ATG4C determined by quantitative RT-PCR. The bar graph indicates the means \pm SEM from three independent experiments. ${ }^{*}, p<0.01$; ${ }^{* *}, p<0.001$ (one-way ANOVA followed by Tukey's Multiple Comparison Test). $\mathbf{k}$ Quantitative analysis of ATG4C expression in CS-derived neurons. (Inset) Western blot images of ATG4C expression in CS-derived neurons. The bar graph indicates the means \pm SEM from five independent experiments. ${ }^{*}, p<0.05 ;{ }^{* *}, p<0.01$ (one-way ANOVA followed by Tukey's Multiple Comparison Test) 
ultrastructural level using EM. Similar to EM results from CS fibroblasts, AVs were accumulated in presynaptic terminals of CS neurons compared to control neurons (Fig. 1e). Autophagic flux was significantly increased in CS neurons compared to control neurons (Fig. 1f-g). These data suggest that autophagy was also upregulated in CS neurons.

Following the previous finding, an examination of how autophagy is upregulated in CS patient-derived fibroblasts and neurons was conducted; previous findings showed that the mRNA level of VPS13B was significantly reduced (approximately 50\%) in CS fibroblasts with heterogenous missense mutations (c.T1239G [p.Y413X] and c.G10333A [p.V3445M]) compared to control fibroblasts (under revision). Therefore, to clarify the role of VPS13B in autophagy, we used the CRISPR/Cas9 genome editing system to disrupt the gene encoding human VPS13B in HeLa cells (CRISPR guide RNA sequence [gRNAs] \#1: 5'-GGTAATTACCATCAATACTA-3'; gRNAs \#2: 5' - AATTGAGGATTCATGTACCA-3') and validated knockout of $V P S 13 B$ by genomic PCR. Figure 1h-i shows the level of LC3-II in VPS13B KO cells was higher in the presence of lysosomal inhibitors than in wild-type HeLa cells, indicating that the loss of $V P S 13 B$ is associated with upregulated autophagic flux, suggesting its potential role in basal autophagy.

To discover autophagy-associated genes responsible for the upregulation of autophagy activity in CS neurons, a transcriptomic analysis for autophagy pathwayrelated genes in CS neurons and control neurons was implemented. In CS neurons, Gene Ontology analysis showed that two processes (autophagosome organization and macroautophagy) were significant. MFN2, $A T G 9 B$, ATP13A2, KIAA1324, MAP1LC3C, and ATG4C genes were upregulated in CS neurons compared to control neurons. To provide further confirmation, quantitative RTPCR, and western blot analyses were performed from control and CS neurons and revealed that mRNA and protein levels of $A T G 4 C$ in CS neurons were higher compared to control neurons (Fig. 1j-k). ATG4 is a cysteine protease required for LC3/gamma-aminobutyric acid receptorassociated protein (GABARAP) processing, which allows for the latter to be conjugated to phosphatidylethanolamine on autophagosomal membranes; a key step in autophagosome biogenesis and recycling [9].

Further examination was undertaken to see whether the gene expression of $L C 3 B$ or GABARAP was affected in CS neurons. However, there was no significant difference in mRNA expression of $L C 3 B$ or GABARAP between control and CS neurons (data not shown). The autophagic flux may be increased, at least partially, by the upregulation of $A T G 4 C$ in CS neurons; however, $V P S 13 B$ 's association with the upregulation of autophagy and CS pathogenesis should be further investigated. The
VPS13 family is associated with various human diseases [10]; in particular, a loss of VPS13A function disrupts autophagic flux and leads to chorea-acanthocytosis $(\mathrm{ChAc})$, a rare neurodegenerative disease with no known cure. The absence of VPS13A or VPS13B causes defective or increased autophagic flux; for this reason, autophagy might be a promising therapeutic target for human diseases associated with VPS13 [2, 11].

\section{Abbreviations}

CS: Cohen syndrome; VPS13B: vacuolar protein sorting 13 homolog B; ATG: autophagy-related gene; GABARAP: gamma-aminobutyric acid receptorassociated protein; LC3: microtubule-associated proteins 1A/1B light chain 3; CRISPR: clustered regularly interspaced short palindromic repeats

\section{Acknowledgments}

Not applicable.

\section{Authors' contributions}

JA Lee, BK Kaang, YK Lee, CS Lim, K Lee, and JH Lee designed the study, analyzed the data, and wrote the paper. YS Lee, SK Lee, S Choi, YH Huh, JH Kwak, and DJ Jang performed the experiments. The author(s) read and approved the final manuscript.

\section{Funding}

This work was supported by National Research Foundation (NRF2017R1D1A3B03030972), the National Honor Scientist Program, the Korea Health Technology R\&D Project (H118C0158), and the Bio \& Medical Technology Development Program of the National Research Foundation (NRF) funded by the Ministry of Science \& ICT (2017M3A9G7073521) to J.-A L.

Availability of data and materials

All data generated or analyzed during this study are included in this published article.

Ethics approval and consent to participate

Not applicable.

\section{Consent for publication}

Not applicable.

\section{Competing interests}

The authors declare that they have no competing interests.

\section{Author details}

${ }^{1}$ Department of Biological Sciences and Biotechnology, Hannam University, 1646 Yuseongdaero, Yuseong-gu, Daejeon 34054, Korea. ${ }^{2}$ Center for Electron Microscopy Research, Korea Basic Science Institute, Daejeon 34133, Korea. ${ }^{3}$ Department of Anatomy, Brain Science \& Engineering Institute, Kyungpook National University School of Medicine, Daegu 41944, Korea. ${ }^{4}$ Department of Physiology, Biomedical Sciences, Neuroscience Research Institute, Seoul National University College of Medicine, Seoul 03080, Korea. ${ }^{5}$ Department of Ecological Science, College of Ecology and Environmental Science, Kyungpook National University, Sangju 37224, Korea. ${ }^{6}$ Department of Life and Nanopharmaceutical Sciences, Department of Oral Microbiology, School of Dentistry, Kyung Hee University, Seoul 02447, Korea. ${ }^{7}$ Department of Biological Sciences, College of Natural Sciences, Seoul National University, Seoul 08826, Korea. ${ }^{8}$ Department of Pharmacology, Wonkwang University School of Medicine, 460 Iksan-daero, Iksan 54538, Korea.

Received: 23 February 2020 Accepted: 28 April 2020

Published online: 06 May 2020

\section{References}

1. Velayos-Baeza A, Vettori A, Copley RR, Dobson-Stone C, Monaco AP. Analysis of the human VPS13 gene family. Genomics. 2004:84(3):536-49.

2. Kaminska J, Kolakowski D. Proteins from Vps13 family: from molecular function to pathogenesis of neurodegenerative disorders. Postepy Biochem. 2018;64(4):275-87 
3. Kim MJ, Lee RU, Oh J, Choi JE, Kim H, Lee K, Hwang S-K, Lee J-H, Lee J-A, Kaang B-K. Spatial learning and motor deficits in vacuolar protein sortingassociated protein 13b (Vps13b) mutant mouse. Experimental Neurobiol. 2019:28(4):485.

4. Seifert W, Kuhnisch J, Maritzen T, Horn D, Haucke V, Hennies HC. Cohen syndrome-associated protein, $\mathrm{COH} 1$, is a novel, giant Golgi matrix protein required for Golgi integrity. J Biol Chem. 2011;286(43):37665-75.

5. Lee YK, Jun YW, Choi HE, Huh YH, Kaang BK, Jang DJ, Lee JA. Development of LC3/GABARAP sensors containing a LIR and a hydrophobic domain to monitor autophagy. EMBO J. 2017;36(8):1100-16.

6. Levine B, Kroemer G. Biological functions of autophagy genes: a disease perspective. Cell. 2019;176(1-2):11-42.

7. Yim WW-Y, Mizushima N. Lysosome biology in autophagy. Cell Discov. 2020; 6(1):6.

8. Azarnia Tehran D, Kuijpers M, Haucke V. Presynaptic endocytic factors in autophagy and neurodegeneration. Curr Opin Neurobiol. 2018:48:153-9.

9. Agrotis A, Pengo N, Burden JJ, Ketteler R. Redundancy of human ATG4 protease isoforms in autophagy and LC3/GABARAP processing revealed in cells. Autophagy. 2019;5(6):976-97.

10. Rodrigues JM, Fernandes HD, Caruthers C, Braddock SR, Knutsen AP. Cohen syndrome: review of the literature. Cureus. 2018;10(9):e3330.

11. Kumar N, Leonzino M, Hancock-Cerutti W, Horenkamp FA, Li P, Lees JA, Wheeler H, Reinisch KM, De Camilli P. VPS13A and VPS13C are lipid transport proteins differentially localized at ER contact sites. J Cell Biol. 2018; 217(10):3625-39.

\section{Publisher's Note}

Springer Nature remains neutral with regard to jurisdictional claims in published maps and institutional affiliations.

Ready to submit your research? Choose BMC and benefit from:

- fast, convenient online submission

- thorough peer review by experienced researchers in your field

- rapid publication on acceptance

- support for research data, including large and complex data types

- gold Open Access which fosters wider collaboration and increased citations

- maximum visibility for your research: over $100 \mathrm{M}$ website views per year

At $\mathrm{BMC}$, research is always in progress.

Learn more biomedcentral.com/submissions 